\title{
Acceleration-based Assistive Strategy to Control a Back-support Exoskeleton for Load Handling: Preliminary Evaluation
}

\author{
Maria Lazzaroni ${ }^{1,2}$, Stefano Toxiri ${ }^{1}$, Darwin G. Caldwell ${ }^{1}$, Sara Anastasi ${ }^{3}$, Luigi Monica ${ }^{3}$, \\ Elena De Momi ${ }^{2}$ and Jesús Ortiz ${ }^{1}$
}

\begin{abstract}
Industrial active exoskeletons have recently achieved considerable interest, due to their intrinsic versatility compared to passive devices. To achieve this versatility, an important open challenge is the design of appropriate control strategies to automatically modulate the physical assistance according to the activity the user is performing.

This work focuses on active back-support exoskeletons. To improve the assistance provided in dynamic situations with respect to state-of-the-art methods, a new strategy making use of the angular acceleration of the user's trunk is presented.

The feasibility and effectiveness of the proposed strategy were tested experimentally on a prototype in a load handling task. The main advantages in terms of assistive torque profiles emerge during the transition phases of the movement (i.e. beginning and end of lowering and lifting) indicating an appropriate adaptation to the dynamics of the execution.

In this preliminary evaluation, the data on peak muscular activity at the spine show promising trends, encouraging further developments and a more detailed evaluation.
\end{abstract}

\section{INTRODUCTION}

Musculoskeletal disorders (MSDs) are the most frequent occupational diseases in many industrial countries [1], with significant impact on quality of life and high costs in lost productivity, sickness absence and disability. Manual handling tasks in industries highly increase workers' probability of developing MSDs [2], resulting from the overloading of workers' body joints [3]. In particular, high risk of developing MSDs is associated with heavy physical work, twisting and bending, frequent lifting of heavy loads and the maintaining of prolonged inconvenient postures [2]. The most commonly reported injuries involve the back, the neck and the upper limbs [4].

As regards MSDs at the back, high risk of injury results from large compressive forces generated on the lumbar spine [5]. During manual material handling, spinal muscles activity, associated with repetitive and prolonged trunk flexion, generates compression on lumbar discs [6], especially, on the L5S1 disc. Higher compression and, thereby, higher incidence of back injuries were significantly associated with the intensity (i.e. the weight of the load handled) and the frequency of the task during work [5].

To reduce the incidence of MSDs in the industry, backsupport exoskeletons were introduced [7] to reduce joints overloading at the back, by reducing the activity of spinal

\footnotetext{
${ }^{1}$ Department of Advanced Robotics, Istituto Italiano di Tecnologia Genova, Italy (corresponding author: maria.lazzaronieit.it).

${ }^{2}$ Department of Electronics, Information and Bioengineering, Politecnico di Milano, Italy.

${ }^{3}$ Italian Workers' Compensation Authority (INAIL)
}

muscles. Muscular activity reduction ranging between 10 and $40 \%$ was associated with the use of passive [7] [8] [9] and active [7] [10] exoskeletons. Correspondingly, the compressive loads on lumbar discs and, therefore, the related risk of injury are expected to decrease.

Active back-support exoskeletons have recently achieved considerable interest in research and in the market, due to their intrinsic potential. Compared to passive devices, active exoskeletons can modulate the assistance during the operation, by means of actuators, and thereby adapt to different tasks and users. The key to adaptability is a control strategy that is able to precisely detect the intention of the user to move and accordingly anticipate and adapt the assistance provided to the user.

Several strategies to modulate exoskeletons assistance have been proposed for manual material handling, focused on assisting the load lifting and lowering. The easiest approach is to manually drive the assistance, using extra joysticks or buttons (e.g., Muscle Suit [11] for back-support). However, the two most prevalent approaches in research and commercial devices are: controllers using mechanically intrinsic signals and controllers using neural signals (e.g., brain activity or muscle activity) [12].

Control driven by mechanically intrinsic signals relies on measures that are intrinsic to the device itself (e.g., gait events, joint angles or forces, segments movements) [12]. For back-support exoskeleton, trunk or legs movements can be registered thanks to Inertial Measurement Units (IMUs) or encoders embedded in the exoskeleton structure. Trunk inclination is usually measured through an IMU and used for gravity compensation (as in Robo-Mate [13] and Hyundai Waist Exoskeleton (H-WEX) [14]). The Active Pelvis Orthosis (APO) [15] uses two encoders and an IMU to detect when the lifting movement starts and then modulates the assistance according to hip angles (from the encoders).

Electromyography-based (EMG) strategies detect user's intention from muscles activity and actuate the device accordingly. Surface EMG signals of flexor and extensor muscle of erector spinae are used to modulate the level and timing of the reference assistive torque for the HAL backsupport exoskeleton [16]. An additional torque compensates the weight of the user.

The Robo-Mate active trunk exoskeleton [13] defines the assistive torque as the combination of both approaches. The inclination-based torque is calculated according to the trunk inclination (measured with an IMU), while the EMGbased torque is defined proportionally to the forearm muscles 
activity, recorded by surface electrodes with a Myo armband (a commercial device that measures surface EMG). Since forearm muscles activity increases when handling a load, the assistance can be automatically adapted to the presence of an external object.

However, considering industrial applications, EMG-based strategies are limited because of the invasiveness of the electrodes and the signal variability (with time, fatigue, sweat and skin artifacts). On the other hand, current exoskeleton strategies using mechanically intrinsic signals are primarily based only on the measure of the static variables of the user's movement (e.g., trunk inclination [13] [14] or hip angle [15]). As a result, the assistance would not adapt to the different phases of a task, i.e. providing the same assistive torque during the descent (lowering) and the ascent (lifting). This feature, thus, limits the physical assistance that can be provided to the user, as a higher help during lifting will results in a higher resistance during lowering, as for passive exoskeletons that employ springs. Furthermore, it has been shown in [17] that the timing of trunk inclination peak may not coincide with the timing of the lumbar moment peak when lifting and lowering an external load. Consequently, an inclination-controlled exoskeleton would not provide the peak assistance in timing with the peak in torque need.

To better assist workers when performing lifting and lowering tasks, we investigated the dynamic variables of the lifting movement (i.e. the velocity and the acceleration of the trunk) and the correlation with the principal factors that cause MSDs (e.g., lumbar moment or lumbar compressive forces). An approximate linear relation was observed between the trunk angular acceleration and the lumbar moment of a user performing lowering and lifting and is presented in Section II. Based on these results, a control strategy for active backsupport exoskeletons is proposed (Section III), accounting for the additional information provided by the angular acceleration of the user's trunk. The strategy was implemented on the Robo-Mate exoskeleton, for which an overview is included. Finally, Section IV provides the experimental evaluation of the new strategy for assisting in lifting and lowering. In Section $\mathrm{V}$ advantages and limitations of the strategy are discussed.

\section{TASK DYNAMICS}

A. The effects of execution speed on user's musculoskeletal system

The extension moment about the lumbar joint indicates the external load applied to the user's musculoskeletal system that causes spine compression and, therefore, increases MSDs risk. However, it remains unclear whether the risk is associated with the cumulative or the peak moment values (or both). Peak moment is one among the five factors identified in [18] that better predicted the risk of developing MSDs during lifting activities. Another study [19] identified the cumulative moment as one of the four major MSDs risk factors.

Several studies have demonstrated how the user's execution speed influences the lumbar moment magnitude. In
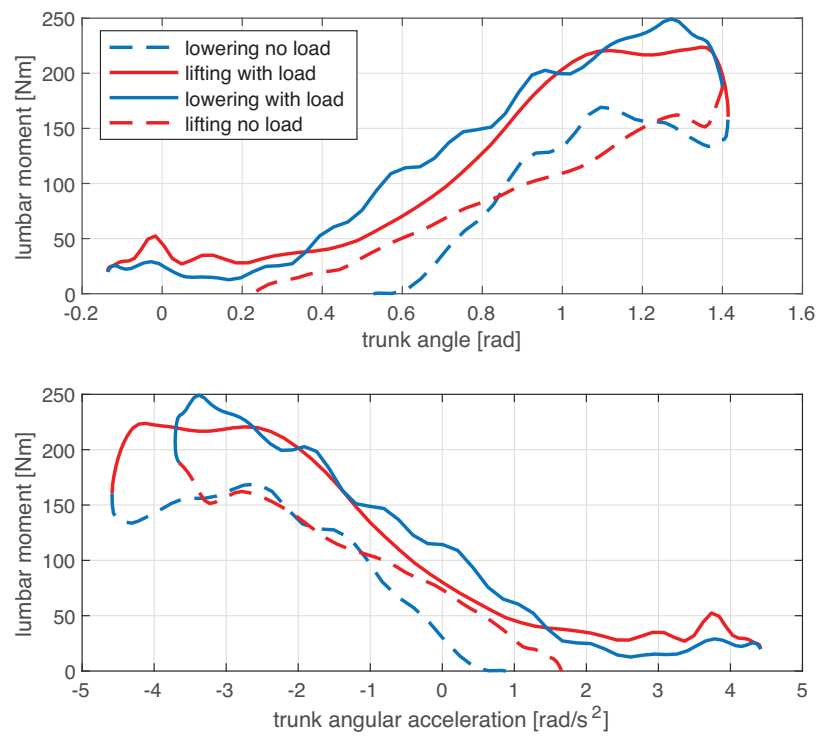

Fig. 1. Lumbar moment correlation with trunk angle (top) and trunk acceleration (bottom) during lifting (red lines) and lowering (blue lines). Solid lines indicate when the subject is holding the external object of 15 $\mathrm{kg}$, while dashed lines indicate when the subject is not holding it. A gap is observable between the solid and dashed lines, showing the lumbar moment dependence on object weight.

[20] experiments determined the effect of the speed on peak and cumulative moment during symmetric lifting tasks. Results showed that, for low lifting speed, the peak load is approximately $18 \%$ lower than for high speed. Conversely, the cumulative load is higher (near $60 \%$ ) at the low lifting speed, due to the longer lifting duration. Interestingly, results showed that peak and cumulative loads change for different execution speeds. Furthermore, a significant increase in the spinal compression with the lifting speed was found in [21]. Another study [22] showed that the peak L5S1 moment linearly increases (with statistical significance) with the increasing lifting speed. Authors suggested to consider the execution speed in a complete analysis of the lifting task, as it is crucial to the moment magnitude. Additionally, among the distinct task phases, differences were observed between lowering and lifting [23]. Both tasks have maximum compression forces that increase with execution speed. However, the compression force increases more with the execution speed when lowering than when lifting.

Therefore, as the speed execution influences the lumbar moment, it would be useful to account for the speed execution in modulating the assistive torque.

\section{B. The effects of dynamics on user's musculoskeletal system}

Based on the evidence that the execution speed influences the lumbar moment during lifting and lowering, we want to expand on the effects of the task dynamics (i.e. the angular velocity and the angular acceleration of the user's trunk) on the musculoskeletal system, in terms of lumbar moment and compression force. The correlation between the user's dynamics and the lumbar moment is accessed in a replicated real scenario of the target tasks. 
Eleven healthy subjects participated in the evaluation of Robo-Mate exoskeleton effectiveness (complete results in [13]). Each subject was instructed to complete a lifting and a lowering task in different conditions, i.e. with and without the exoskeleton. Each task started in an upright position, then the subject bend over, reach and grasp a box of $15 \mathrm{~kg}$ from the handles and take it up to an upright position. After, the subject bent over once more to place the box and go back to an upright posture. No instructions on a specific execution speed were given.

Full-body 3D kinematics and ground reaction forces were integrated with a dynamic 3D biomechanical model [24] to calculate the net moment $M_{L 5 S 1}$ at the L5S1 intervertebral disc via inverse dynamics. To evaluate the correlation between the user's dynamics and the computed moment, we investigated the tasks executed without the exoskeleton. An approximate linear relation was observed both with the trunk angle and the trunk angular acceleration. This is illustrated in Fig. 1 for one subject, as results across all subjects showed high deviations but a similar trend. The gap observable between the solid (with load) and dashed lines (no load) shows the influence of the external object on the lumbar extension moment.

These results include the three key factors that affect lumbar moment: (a) the inclination of the upper body, (b) the mass of the objects lifted and (c) the angular acceleration of the upper body. An exoskeleton that focuses on reducing the overloading in the lumbar joint, has to consider each of these factors.

Control strategies found in the literature have considered the trunk inclination, velocity and the external load weight for control purpose (as indicated in Section I for [14] [15] [13]). However, the trunk angular acceleration was never contemplated for the assistance, despite the evidence of its influence on lumbar load during lifting and lowering. Using the angular acceleration for modulating the assistive torque may help overcome limitations of existing strategies and further improve effectiveness, usability and users' acceptance.

\section{ACCELERATION-BASED ASSISTIVE STRATEGY}

We implemented an assistive strategy on the Robo-Mate exoskeleton able to modulate the assistance accordingly to the user's trunk angular acceleration, based on the results presented in Section II-B (i.e. the trunk angular acceleration correlation with the lumbar moment). An overview of the Robo-Mate prototype is included first, and then the details of the control strategy implemented are described.

\section{A. Exoskeleton Prototype}

Robo-Mate is an active back-support exoskeleton specifically designed to assist workers while performing heavy objects manipulation [25]. It focuses on reducing lumbar overload and, as a consequence, the risk of developing MSDs. The prototype is shown in Fig 2. The physical attachments consist of a waist band, custom Velcro-bands used to fix the leg exoskeleton links to the thighs and
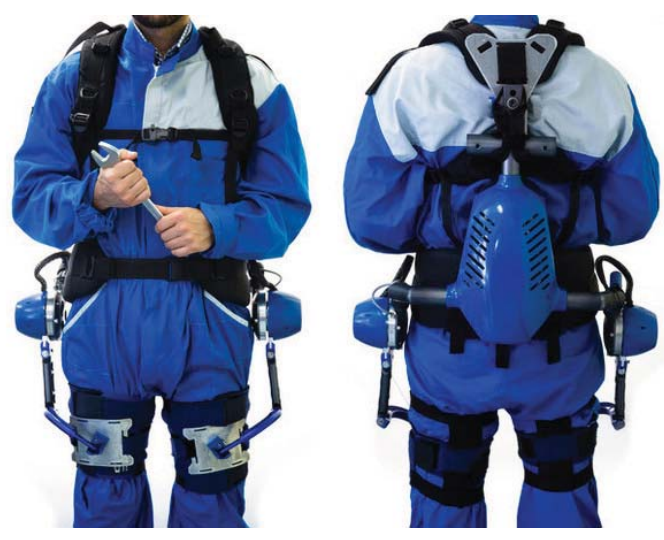

Fig. 2. The Robo-Mate exoskeleton prototype.

shoulder straps. Two electrical torque-controlled actuators are placed at the hip joints (one on each side), approximately aligned with hip flexion-extension axes. They generate a torque in the sagittal plane between the user's torso and thighs. This torque results in assistive forces transmitted to the user by means of the physical attachments. Five passive degrees of freedom endowed in the leg links and a spherical joint connecting the shoulder straps and the rigid frame (i.e. three degrees of freedom) ensure the unhindered movement of the user.

The control scheme is structured on two levels (Fig. 3). The mid-level (i.e. the control strategy) integrates different signals coming from the human (trunk angle $\theta_{h}$ and trunk acceleration $\left.\ddot{\theta}_{h}\right)$ and regulates the related gain $\left(K_{\text {incl }}\right.$ and $K_{a c c}$ ). Accordingly, it generates a reference torque signal $\tau_{\text {ref }}$ (with appropriate timing and magnitude) that is sent to the left and the right actuators. At the low level, each actuator follows this reference signal with a closed-loop torque controller, regulating the output torque $\tau_{\text {out }}$ provided to the user.

Different control strategies to modulate Robo-Mate assistance have been proposed [13]. According to each strategy working concept, a reference torque for actuators is generated to allow the free movement of the users or to assist the movement addressing their requirements.

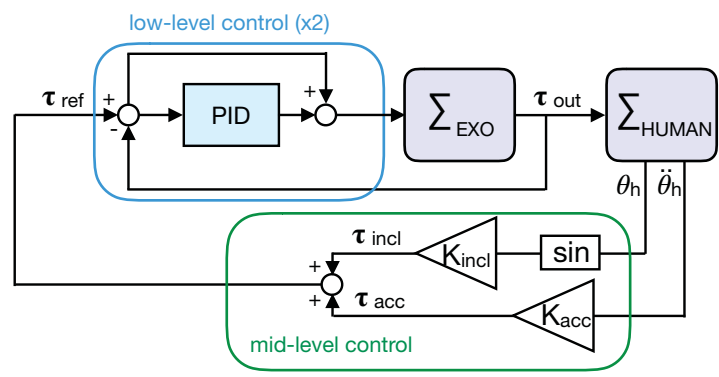

Fig. 3. Block diagram representation of the two levels control system. The exoskeleton $\Sigma_{\text {EXO }}$ (as an admittance) and the human $\Sigma_{\text {HUMAN }}$ (as an impedance) are mechanically interconnected. 


\section{B. Dynamic control strategy}

An xSens MTw IMU (Xsens Technology) was attached to the user's trunk (approximately at the sternum) to measure the trunk angular velocity $\dot{\theta}_{h}$. The trunk angular acceleration $\ddot{\theta}_{h}$ is obtained by differentiating and filtering the angular velocity on the sagittal plane. For the purpose of control, the acceleration is low-pass filtered with a cut-off frequency set to $1 \mathrm{~Hz}$, chosen empirically as a trade-off between signal delay and signal noise.

The torque reference signal $\tau_{a c c}$ related to the user's trunk acceleration is then computed according to the following formula:

$$
\tau_{a c c}=-K_{a c c} \ddot{\theta}_{h}
$$

where $K_{a c c}$ is the control gain that can be adjusted for each user and task to suit individual preferences (e.g., comfort and perceived pressure) and task conditions. Loosely speaking, this acceleration-based torque may be seen as compensating for part of the inertia of the user's upper body.

$\tau_{a c c}$ is added to the inclination-based strategy (referred to as inclination strategy), and already implemented on the Robo-Mate exoskeleton [13]. The inclination strategy assists users to partially compensate for the effect of gravity on lumbar compression, due to exoskeleton and their own upper body weight during flexion. The assistive torque $\tau_{\theta}$ is defined proportionally to the sine of the user's trunk angle $\theta$ in the sagittal plane (acquired with an xSens MTi-30 AHRS IMU (Xsens Technology) embedded on the exoskeleton back structure):

$$
\tau_{\text {incl }}=K_{\text {incl }} \sin \left(\theta_{h}\right)
$$

where $K_{\text {incl }}$ is the inclination assistance gain.

By summing up $\tau_{i n c l}$ and $\tau_{a c c}$ (inclination-based and acceleration-based) it is possible to assist the user according to his/her statics and dynamics. The reference torque $\tau_{\text {dynamic }}$, thus, is computed with the following formula:

$$
\tau_{\text {dynamic }}=\tau_{\text {incl }}+\tau_{a c c}=K_{\text {incl }} \sin \left(\theta_{h}\right)-K_{a c c} \ddot{\theta}_{h}
$$

This assistance can be referred to as dynamic strategy, for which the static (i.e. $\tau_{i n c l}$ ) and the dynamic (i.e. $\tau_{a c c}$ ) contribution can be differently scaled, adjusting the related control gains.

\section{EXPERIMENTAL EVALUATION}

We devised an experimental protocol to assess how the dynamic control strategy changes the assistance provided to the user during lowering and lifting tasks. To evaluate the effectiveness of the strategy, we assess whether the muscular effort during the task execution is reduced, taking as references for the comparison the same task executed without the exoskeleton and with the exoskeleton with the previous strategy (i.e. inclination strategy).

\section{A. Methods}

In the experiment, seven healthy subjects performed the lifting and the lowering of an external weight of $5 \mathrm{~kg}$ and $10 \mathrm{~kg}$, repeated at three different speed: fast, normal and slow. No instructions on a specific lifting technique (i.e., stoop or

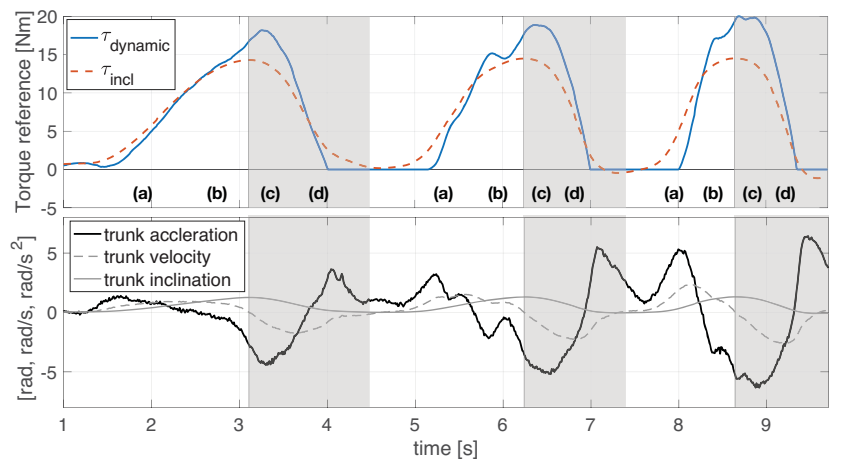

Fig. 4. At the top, reference torque generated by the dynamic strategy (blue) with respect to the torque generated by inclination strategy (red dashed lines). The different phases of the task are highlighted: beginning (a) and end (b) of lowering, beginning (c) and end (d) of lifting (grey background). From left to right the task execution speed is increasing. Trunk inclination, angular velocity and angular acceleration are displayed at the bottom.

squat) was given. The task was executed in three different conditions:

- without the exoskeleton: no-exo

- with the exoskeleton: inclination strategy (Eq. 2) with $K_{\text {incl }}=15$

- with the exoskeleton: dynamic strategy (Eq. 3) with $K_{\text {incl }}=15$ and $K_{a c c}=1$.

To allow comparison between subjects, we have empirically selected the values for the gains $K_{\text {incl }}$ and $K_{a c c}$, instead of adjusting them to the subjects' individual preferences and body characteristics. The no-exo condition was performed first in all cases, while the order of the two conditions with the different strategies was randomized.

An illustration of the reference torques generated by the two strategies (computed with Eq. 2 and 3, respectively) is provided in Fig. 4 for one subject, together with the trunk inclination (measured with the IMU mounted on the exoskeleton structure), angular velocity (measured with the IMU attached to the user's trunk) and angular acceleration (obtained by deriving the angular velocity) of the user during the execution of the task. The task was performed at increasing execution speed. While the inclination strategy (red dashed lines) follows the trunk inclination (grey dashed line, at the bottom), the assistance provided by the dynamic strategy (blue) is a combination of both the trunk inclination and the trunk angular acceleration with negative sign (black, at the bottom).

Differences between the reference torques $\tau_{\text {incl }}$ and $\tau_{\text {dynamic }}$ can be observed in terms of timing and magnitude. As expected, when no significant acceleration occurs (i.e. low speed, on the left) the two reference torques are comparable. The inclination strategy, thus, is appropriate for this specific case. However, when the movement is performed at higher speeds (on the right), there are substantial differences between the two strategies. The dynamic strategy follows the angular acceleration of the user's trunk, that reflects the will of the subject to move in a certain direction. In particular, the dynamic strategy generates a lower assistive torque in 




Fig. 5. Peaks average and standard deviation of the iliocostalis spinal muscle across all subjects. Data are divided for object mass of $5 \mathrm{~kg}$ at the top and object mass of $10 \mathrm{~kg}$ at the bottom. The bars indicate the three condition: no-exo (yellow), inclination control strategy (red) and dynamic control strategy (blue). Data do not show statistical significance. From left to right the execution speeds are decreasing: fast, normal and slow

correspondence with the beginning of lowering (a) and the end of lifting (d), as the acceleration contribution (negative sign) is subtracted from the total torque. By contrast, a greater assistance is provided between the end of lowering (b) and the beginning of lifting (c) when the acceleration reaches the negative peak.

To evaluate the strategies effectiveness, surface EMG electrodes (BTS FREEEMG, BTS Bioengineering) was used to measure the activity of spinal muscle (iliocostalis), following SENIAM guidelines. The measured EMG signals were high-pass filtered (2rd-order Butterworth filter, $20 \mathrm{~Hz}$ cut-off frequency), rectified and low-pass filtered (2rd-order Butterworth filter, $4 \mathrm{~Hz}$ cut-off frequency) to obtain the envelope. Finally, EMG signals were normalized to the maximal voluntary contraction (MVC) performed against manual resistance during a preliminary procedure. For each condition (no-exo, inclination, dynamic), object mass (5 and $10 \mathrm{~kg}$ ) and execution speed (fast, normal and slow) the peak activity was extracted as a measure of the muscular effort requested to the subjects to execute the task. Data analysis was performed in Matlab 2018a (The Mathworks, Natick, USA).

\section{B. Results}

Results (peaks average and standard deviation across all subjects) are shown in Fig. 5, for each condition (no-exo (yellow), inclination (red), dynamic (blue)), object mass (5 at the top and $10 \mathrm{~kg}$ at the bottom) and execution speed (from left to right: fast, normal and slow) With respect to the noexo condition, peak activity is reduced by the exoskeleton with both strategies and for both loads. Moreover, a greater reduction is observed with the dynamic strategy. The data do not show statistical significance (discussion in Section V).

\section{DISCUSSION}

With respect to the inclination control strategy, the main advantage of the dynamic strategy emerges during the transition phases of the movement (i.e. beginning and end of lowering (a) (b) and lifting (c) (d)) and especially for high execution speed (Fig. 4). In fact, with the acceleration, it is possible to follow the intention of the subject, by detecting when the velocity increases or decreases, that is considered to be representative of the will of the subject to start or to slow down the movement in a certain direction.

Specifically, the dynamic strategy generates a lower torque in correspondence with the beginning of lowering (a). Indeed, in this phase of the task, a higher assistance may be perceived as hindering the user in bending over. By contrast, a greater assistance is provided at the end of lowering (b), when the user is decelerating his descent, for helping him to stop. Subsequently, during lifting, the dynamic strategy provides an additional assistance at the beginning (c), when the subject grasps the object and accelerates upwards. Indeed, at the beginning of lifting an additional assistance is desirable as the lumbar moment reaches its maximum value (as provided in [17]). The assistance is reduced at the end of lifting to help the user to reach the upright position, by decreasing the force that is pushing him backwards.

With respect to previous studies employing the user angular velocity [14] [15], the same effect in terms of reducing the assistance that hinders the user at the beginning of lowering (a) is obtained. However, an unwanted effect would result at the end of lowering (b), as a lower assistance would be provided to the user, requesting him a greater effort to decelerate the descent. The same behavior would emerge during lifting phase. The movement upward would be facilitated (c), while a greater effort would be asked to the user at the end of lifting (d) to stop. Indeed, force augmentation achieved by positive feedback employing velocity (as the one presented in [14]) was proved to decrease the user's stability [26].

Even though promising results were observed in terms of EMG peak activity reduction using the new control strategy, the data do not show statistical significance (oneway ANOVA), which may be due to the limited number of participants and repetitions of the task. Future works will focus on a more detailed evaluation of the proposed strategy, with a larger number of subjects and object weights, that will include EMG measurements, biomechanical analysis, and well-defined questionnaires as proposed in [27].

The main limitation of the presented strategy is that an additional IMU attached to the user's trunk has to be included. In fact, on preliminary pilots using the onboard IMU (already embedded on the exoskeleton back structure) a substantial delay was observed between the angular acceleration signal and the actual movement of the user. This may be due to the imperfect coupling between exoskeleton structure and user's body, which allows for relative movement between the two.

A further limitation regards the angular acceleration signal, that is an approximate and noisy estimation obtained by differentiating and filtering the angular velocity. In particular, 
the design of filters requires a trade-off between signal delay and noise. Moreover, overestimating the acceleration can lead to feedback inversion and instability [28]. To avoid excessive acceleration-based torque, the magnitude of the acceleration gain $K_{a c c}$ should be considered carefully. As this controller is compensating the inertia, the $K_{a c c}$ value must be chosen as to not overcompensate. Future works will also address the tuning of the gain $K_{a c c}$ in order to adjust it to subjects individual preferences (e.g., comfort and perceived pressure), body characteristics, and task conditions.

Integration with already existing or new control strategies will be considered. Potential benefits may be achieved by adding an assistive torque that provides the assistance based on the weight of the external load handled (e.g., the EMGbased strategy implemented on Robo-Mate exoskeleton).

\section{CONCLUSIONS}

A new control strategy for an active back-support exoskeleton was presented based on both the inclination and the angular acceleration of the user's trunk. By supporting in accordance with the user's trunk angular acceleration, this strategy addresses one of the key factors that affect lumbar moment. Furthermore, trunk acceleration is considered representative of the user's intention to start or to slow down the movement in a certain direction. This allows overcoming some practical limitations of existing strategies. In fact, using the acceleration, the assistance provided to the user can be modulated for the different phases of a task (e.g., lowering and lifting). By contrast, passive and active exoskeletons based only on static information of the user's movement (e.g., trunk inclination, hip angle) would not adjust to task dynamics such as varying speed.

\section{REFERENCES}

[1] L. Punnett and D. H. Wegman. Work-related musculoskeletal disorders: the epidemiologic evidence and the debate. Journal of electromyography and kinesiology, 14(1):13-23, 2004.

[2] J Zurada. Classifying the risk of work related low back disorders due to manual material handling tasks. Expert Systems with Applications, 39(12):11125-11134, 2012.

[3] S. Kumar. Theories of musculoskeletal injury causation. Ergonomics, 44(1):17-47, 2001.

[4] J. C. Davies, G. J. Kemp, S. P. Frostick, C. E. Dickinson, and J. McElwaine. Manual handling injuries and long term disability. Safety science, 41(7):611-625, 2003.

[5] P. Coenen, V. Gouttebarge, A. S. A. M. Van Der Burght, J. H. van Dieën, M. H. W. Frings-Dresen, A. J. van der Beek, and A. Burdorf. The effect of lifting during work on low back pain: a health impact assessment based on a meta-analysis. Occup Environ Med, pages oemed-2014, 2014.

[6] N. P. Reeves and J. Cholewicki. Modeling the human lumbar spine for assessing spinal loads, stability, and risk of injury. Critical Review in Biomedical Engineering, 31(1\&2), 2003.

[7] M. P. de Looze, T. Bosch, F. Krause, K. S. Stadler, and L. W. OSullivan. Exoskeletons for industrial application and their potential effects on physical work load. Ergonomics, 59(5):671-681, 2016.

[8] T. Bosch, J. van Eck, K. Knitel, and M. de Looze. The effects of a passive exoskeleton on muscle activity, discomfort and endurance time in forward bending work. Applied ergonomics, 54:212-217, 2016.

[9] C. A. Lotz, M. J. Agnew, A. A. Godwin, and J. M. Stevenson. The effect of an on-body personal lift assist device (plad) on fatigue during a repetitive lifting task. Journal of Electromyography and Kinesiology, 19(2):331-340, 2009
[10] K. Miura, H. Kadone, M. Koda, T. Abe, H. Kumagai, K. Nagashima, K. Mataki, K. Fujii, H. Noguchi, T. Funayama, et al. The hybrid assistive limb (hal) for care support successfully reduced lumbar load in repetitive lifting movements. Journal of Clinical Neuroscience, 53:276-279, 2018

[11] Y. Muramatsu, H. Umehara, and H. Kobayashi. Improvement and quantitative performance estimation of the back support muscle suit. In Engineering in Medicine and Biology Society (EMBC), 2013 35th Annual International Conference of the IEEE, pages 2844-2849. IEEE, 2013.

[12] J. R. Koller, C. D. Remy, and D. P. Ferris. Biomechanics and energetics of walking in powered ankle exoskeletons using myoelectric control versus mechanically intrinsic control. Journal of neuroengineering and rehabilitation, 15(1):42, 2018.

[13] S. Toxiri, A. S. Koopman, M. Lazzaroni, J. Ortiz, V. Power, M. P. de Looze, L. O'Sullivan, and D. G. Caldwell. Rationale, implementation and evaluation of assistive strategies for an active back-support exoskeleton. Frontiers in Robotics and AI, 5:53, 2018.

[14] H. K. Ko, S. W. Lee, D. Koo, I. Lee, and D. J. Hyun. Waist-assistive exoskeleton powered by a singular actuation mechanism for prevention of back-injury. Robotics and Autonomous Systems, 2018.

[15] B. Chen, L. Grazi, F. Lanotte, N. Vitiello, and S. Crea. A real-time lift detection strategy for a hip exoskeleton. Frontiers in neurorobotics, 12:17, 2018.

[16] H. Hara and Y. Sankai. Development of hal for lumbar support. In SCIS \& ISIS SCIS \& ISIS 2010, pages 416-421. Japan Society for Fuzzy Theory and Intelligent Informatics, 2010.

[17] A. S. Koopman, S. Toxiri, M. P. de Looze, I. Kingma, and J. H. van Dieën. Effects of an inclination-controlled active spinal exoskeleton on spinal compression forces. International Symposium on Wearable Robotics, pages 505-509, 2018.

[18] W. S. Marras, S. A. Lavender, S. E. Leurgans, F. A. Fathallah, S. A. Ferguson, W. Gary Allread, and S. L. Rajulu. Biomechanical risk factors for occupationally related low back disorders. Ergonomics, 38(2):377-410, 1995.

[19] R. W. K. Norman, R. P. Wells, P. W. Neumann, J. W. Frank, H. S. Shannon, and M. S. Kerr. A comparison of peak vs cumulative physical work exposure risk factors for the reporting of low back pain in the automotive industry. Clinical biomechanics, 13(8):561-573, 1998.

[20] K. O. Greenland, A. S. Merryweather, and D. S. Bloswick. The effect of lifting speed on cumulative and peak biomechanical loading for symmetric lifting tasks. Safety and health at work, 4(2):105-110, 2013.

[21] K. P. Granata and W. S. Marras. An emg-assisted model of trunk loading during free-dynamic lifting. Journal of biomechanics, 28(11):1309-1317, 1995.

[22] C. Bush-Joseph, O. Schipplein, G. B. J. Andersson, and T. P. Andriacchi. Influence of dynamic factors on the lumbar spine moment in lifting. Ergonomics, 31(2):211-216, 1988.

[23] K. G. Davis, W. S. Marras, and T. R. Waters. Evaluation of spinal loading during lowering and lifting. Clinical Biomechanics, 13(3):141-152, 1998.

[24] I. Kingma, M. P. de Looze, H. M. Toussaint, H. G. Klijnsma, and T. B. M. Bruijnen. Validation of a full body 3-d dynamic linked segment model. Human Movement Science, 15(6):833-860, 1996.

[25] S. Toxiri, J. Ortiz, J. Masood, J. Fernández, L. A. Mateos, and D. G. Caldwell. A wearable device for reducing spinal loads during lifting tasks: Biomechanics and design concepts. In Robotics and Biomimetics (ROBIO), 2015 IEEE International Conference on, pages 2295-2300. IEEE, 2015

[26] J. A. Norris, A. P. Marsh, K. P. Granata, and S. D. Ross. Positive feedback in powered exoskeletons: Improved metabolic efficiency at the cost of reduced stability? In ASME 2007 International Design Engineering Technical Conferences and Computers and Information in Engineering Conference, pages 1619-1626. American Society of Mechanical Engineers, 2007.

[27] M. Lazzaroni, S. Toxiri, J. Ortiz, E. De Momi, and D. G. Caldwell. Towards standards for the evaluation of active back-support exoskeletons to assist lifting task. In Sixth international Congress of Bioengineering, 2018.

[28] A Calanca, R. Muradore, and P. Fiorini. Impedance control of series elastic actuators: Passivity and acceleration-based control. Mechatronics, 47:37-48, 2017. 